\title{
EL DISEÑO DEL GANAL DE DISTRIBUCIÓN INTERNACIONAL COMO VENTAJA DIFERENCIAL DE MARKETING: CASO TOYOTA
}

\author{
DESIGN INTERNATIONAL DISTRIBUTION CHANNEL DIFFERENTIAL \\ AS MARKETING ADVANTAGE: IF TOYOTA
}

\author{
Raúl Moisés Camargo Hermosilla* \\ rcamargoh@unmsm.edu.pe
}

[RECEPCIÓN: MARZO DE 2015 / CONFORMIDAD: ABRIL DE 2015]

\begin{abstract}
RESUMEN
Todo producto percibido como distinto de los demás es un "producto diferente", aunque en la práctica sea similar. Siguiendo con este enfoque que ubica a la diferenciación como una variable clave para competir, las ventajas físicas y funcionales son muy necesarias para participar en un mercado competitivo, pero no suficientes para ganar la fidelidad de los usuarios.

En la generalidad de las industrias manufactureras, la distribución y el respaldo al producto son activos estratégicos subvalorados. Hoy en día, la excelencia en la ingeniería, la eficiencia en la manufactura y la calidad están convirtiéndose rápidamente en elementos que se dan por sentados.
\end{abstract}

Palabras clave: Canal de distribución internacional.

\begin{abstract}
Any product perceived as different from others is a "different product", although in practice it is similar. Following this approach that places differentiation as a key variable to compete, Physical and functional advantages are needed to participate in a competitive market, but not enough to win the loyalty of users.

In the majority of manufacturing, distribution and product support strategic assets are undervalued. Today, excellence in engineering, manufacturing efficiency and quality are quickly becoming elements that are taken for granted.
\end{abstract}

Keywords: International Distribution Channel.

\footnotetext{
Profesor asociado de la Facultad de Ciencias Administrativas; magíster en Administración de la Educación por la Universidad de Lima. El presente artículo es un resumen del Informe Final del Proyecto SIN-SIN 2014: El diseño del canal de distribución internacional como ventaja diferencial del marketing: caso Toyota.
} 


\section{INTRODUCCIÓN}

La evolución que ha tenido los canales de distribución comercial, a partir de la segunda mitad del siglo XX, ha configurado un panorama complejo, diverso y muy diferente al que se conocía anteriormente. Por entonces estas actividades eran consideradas como una intermediación entre el fabricante y el consumidor; en la actualidad la distribución comercial se han tornado en protagonista esencial de la actividad económica. Participa como generador de empleo, contribuye al PBI creando utilidad para los consumidores, se ha convertido en el sector clave para entender la economía del siglo XXI.

La necesidad de poner en contacto a los fabricantes con los lugares de consumo justifica la existencia de los canales de distribución. La utilidad que aporta la distribución comercial a la economía se concreta en la superación de las distancias existentes entre fabricante y consumidor. Los canales de distribución, como vías por los que circulan los productos, realizan una serie de funciones necesarias para que el producto deseado por el consumidor final esté disponible, toda vez que añaden valor a la mercancía producidas en la fábrica.

\section{OBJETIVOS}

1. Identificar las distintas estrategias disponibles para diseñar canales de distribución comercial internacional.

2. Identificar las variables pertinentes que intervienen en el diseño de un canal distribución comercial internacional que generen ventajas diferenciales.

3. Identificar la estrategia de canal de distribución comercial de Toyota que le otorga una ventaja diferencial en el mercado internacional frente a sus competidores.

\section{JUSTIFICACIÓN}

La economía del Perú se ha basado tradicionalmente en la explotación, procesamiento y exportación de recursos naturales, principalmente mineros, agrícolas y pesqueros. No obstante, en los últimos años se observa una muy importante diversificación y un notable crecimiento en servicios e industrias ligeras. Exportaciones peruanas alcanzaron un récord de US\$ 45,726 millones en el 2011. Las exportaciones no tradicionales supera- ron los US\$10,158 millones, lo que representó un aumento de $32 \%$, todo un récord en los productos con mayor valor agregado", lo que evidencia que el Perú se fortalece cada vez mas en las actividades del comercio internacional, para ello requiere adquirir competencias de marketing que le permitan competir con éxito en este mercado.

Lo principal para una empresa es "ser diferente" en un mundo globalizado y altamente competitivo donde existe un exceso de oferta. Lo importante es diferenciarse de otros muchos que ofrecen cosas iguales o muy parecidas, así el diseño de un canal de distribución comercial deviene en una estrategia diferenciadora.

Los resultados de la investigación contribuirán a difundir entre los ejecutivos de marketing de las empresas exportadoras, además de sensibilizarlos, sobre lo importante que es administrar y gestionar los canales de distribución internacional como una estrategia para obtener una ventaja diferencial y competir en el mercado con éxito.

\section{HIPÓTESIS}

Mediante el diseño de canales de distribución comercial, considerando las variables pertinentes, se obtiene una ventaja diferencial de marketing para las empresas que operan en el mercado internacional.

\section{META ESPECÍFICA}

Comprobar empíricamente que el adecuado diseño del canal de distribución comercial internacional otorga una ventaja diferencial de marketing a la empresa.

\section{MARCO TEÓRICO DEL TRABAJO}

Los canales de distribución se estudian en el contexto de la distribución comercial; por lo tanto, iniciamos su estudio analizando algunos conceptos: distribución comercial, canal de distribución, las funciones del canal de distribución, la estructura de los canales de distribución y su clasificación. Estos conceptos nos proporcionarán una visión completa de la teoría que nos servirá para el estudio de la presente investigación.

\section{Conceptos de distribución comercial}

Se han identificado dos conceptos relevantes y de actualidad sobre la distribución comercial que a continuación se presentan: 
Los autores Vázquez y Trespalacios (2006) en señalan: «La distribución comercial se concibe como un conjunto de actividades cuyo propósito es posibilitar el uso final de los bienes y servicios producidos por los fabricantes. La distribución comercial abarca el conjunto de actividades necesarias para colocar los bienes y servicios producidos por las industrias a disposición de los consumidores finales para satisfacer sus necesidades y deseos» (p.5).

Otro concepto hallado es el que proponen los autores Miquel et al. (2008), quienes desarrollan una propuesta más amplia: «La distribución se configura como un puente entre producción y consumo. Un puente que tiene repercusiones económicas y sociales. Desde el punto de vista económico, la distribución es el eje organizador de los intercambios. La división del trabajo requiere de una actividad distributiva que incremente el valor de los bienes y servicios producidos a través de la preparación y transporte, desde las zonas de producción a los centros de consumo. Desde el punto de vista social, la distribución supone una gran fuente de generadora de empleo. Se trata de un sector de trabajo intensivo, y desempeña un papel importante como refugio de la emigración rural y como estabilizador de las fluctuaciones de la economía» (p. 16).

Resumiendo, la distribución comercial viene a ser el conjunto de actividades relacionadas con la esencia del marketing (intercambio), tendientes a satisfacer necesidades de los consumidores; actividades cuyo propósito es conectar a los productores con los consumidores finales, que conllevan repercusiones económicas y sociales.

\section{Conceptos de canal de distribución}

En líneas precedentes afirmábamos que los canales de marketing se explican en el contexto de la distribución comercial; desde esta perspectiva se presentan varia definiciones respecto de los canales de distribución:

Pelton y Strutton (1999) definen el canal de distribución así: «Un [canal de marketing]puede definirse como un ordenamiento de relaciones de intercambio que crean valor para el cliente en la adquisición, consumo y disposición de productos y servicios» (p.10).
Miquel et al. (2008), sobre los canales de distribución, sostienen: «El canal de distribución está constituido por la trayectoria que ha de seguir un bien o un servicio desde su punto de origen o producción hasta su consumo, y además, por el conjunto de personas y/o entidades que permiten la realización de las tareas correspondientes a lo largo de dicha trayectoria» (p. 57).

Los autores Vázquez y Trespalacios (2009) definen el canal de distribución como sigue: «Se entiende en la práctica por canal de distribución el conjunto de organizaciones -fabricantes, mayoristas, minoristas y u otros agentes comerciales- que reúnen sus fuerzas para entregar los bienes a los usuarios industriales o consumidores finales» (p. 9).

El término canal de marketing se utilizó para describir la existencia de un canal de comercio que establece un puente entre productores y consumidores. Los primeros autores compararon los canales de marketing con caminos a través de los cuales los bienes o materiales podrían pasar de los productores a los consumidores. Este movimiento de productos y servicios solo puede ser posible a través del proceso de intercambio que es la finalidad del marketing. Así, los canales de marketing facilitan el proceso de intercambio, cualquier conexión entre individuos y/u organizaciones que permite o contribuya a la realización de un intercambio es un canal de marketing.

\section{Estructura de los canales de distribución}

La estructura de los canales es variada pudiendo existir un canal con un componente único, fabricante o productor, que asume todas las funciones y vende la mercancía directamente a los compradores finales. En otros muchos casos intervienen intermediarios comerciales, junto con otros agentes externos, produciéndose, de hecho, un reparto funcional entre todos ellos.

Se distingue, en este sentido, dos tipos de estructuras en el canal de distribución: la estructura básica y la estructura superficial

- Estructura básica. Formada por todas aquellas organizaciones que en algún momento llegan a tener posesión y/o propiedad del producto. Cada una de ellas forma una etapa de la estructura bá- 
sica. Por ejemplo, el canal de distribución compuesto por fabricantes, mayoristas y minoristas.

- Estructura superficial. El resto de las organizaciones o agentes intervinientes en la tarea de la distribución comercial, que facilitan y apoyan la labor de la estructura básica y que en la mayoría de las ocasiones no tiene posesión y/o propiedad de los productos comercializados. Por ejemplo, bancos, empresas de seguros y transporte. (Vázquez y Trespalacios 2006, p. 18-19)

\section{Clasificación de los canales de distribución}

Siguiendo los criterios habituales de los manuales de texto sobre la materia, se clasifican los canales de distribución con arreglo a dos referencias: la longitud y el grado de unión o vinculación entre los miembros integrantes del sistema.

\section{a) Según longitud del canal de distribución:}

- Canal indirecto, que puede ser canal corto, cuando utilizan un único intermediario que suele ser un minorista; y canal largo, cuando el circuito cuenta con dos o más intermediarios en distintos niveles.

- Canal directo. No interviene intermedio alguno.

b) Según el grado de vinculación existente

- Canal convencional. Se caracteriza por presentar una vinculación mínima entre los participantes en la distribución de los productos, limitada a la compra-venta.

- Sistemas verticales de distribución. Se caracteriza por una mayor coordinación entre los miembros del canal.

- Sistema vertical corporativo.

- Sistema vertical contractual.

- Sistema vertical administrado.

- Sistemas horizontales de distribución. (Vázquez y Trespalacios 2006, p. 19-20).

\section{Modo de entrada al mercado internacional}

Las opciones para un agente de marketing van desde asumir la actividad de distribución completa (al establecer sus propias filiales y marketing directamente hasta el usuario final) hasta depender de intermediarios para la distribución del producto. Se presentan tres alternativas: (i) los intermediarios localizados físicamente en el país de origen del fabricante; (ii) los intermediarios localizados físicamente en los países extranjeros; $\mathrm{y}$ (iii) finalmente, los intermediarios afiliados al gobierno.

Algunos tipos de intermediarios locales disponibles son: tienda de venta al público de los fabricantes, vendedores globales, compañías comerciales exportadoras, agentes locales, mayoristas para la exportación, intermediarios de exportación, intermediarios de países extranjeros.

La variedad de vendedores y agentes intermediarios en la mayoría de los países es similar a la que existe en el país de origen. Algunos de los intermediarios de países extranjeros son representantes de fabricantes y distribuidores extranjeros, así como agentes representantes y compradores, concesionarios que tienen una relación continua con el fabricante (Cateora y Graham 2006, pp. 415-421).

\section{DISEÑO DE LA INVESTIGACIÓN, MÉTODO Y TÉCNICAS UTILIZADAS}

El método utilizado ha sido el estudio de caso, la técnica empleada ha sido la revisión documental, mediante la revisión de archivos digitales y realización de entrevistas en profundidad, mediante un análisis cualitativo. Se identificaron y clasificaron las variables estratégicas para el diseño de canales de distribución internacional, como factores explicativos y contraste con la realidad.

\section{POBLACIÓN}

El objeto de estudio es la corporación transnacional Toyota, cuyo perfil se presenta a continuación (Toyota Motor Corporation s.f. a).

$\begin{array}{ll}\text { Nombre de la compañía } & \text { :Toyota Motor Corporation } \\ \text { Presidente } & \text { : Akio Toyoda } \\ \text { Oficina central } & \text { : Toyota City, Aichi Prefecture 471- } \\ \text { 8571, Japan, phone: (0565) 28-2121 } \\ \text { Fecha de fundación } & : \text { Agosto 28, 1937 } \\ \text { Capital } & : 397.05 \text { billion yen (as of May 2014) } \\ \text { Número de empleados } & : 338,875 \text { (as of March 31, 2014) }\end{array}$

Misión, Visión, Principios, Valores

En esta sección revisamos la declaración de la misión, visión, principios y valores de la cor- 
poración. Cuya información se ha obtenido de la página web de la corporación:

\section{Misión}

«Ofrecer a nuestros clientes automóviles la prestigiosa marca Toyota, basada en una entrega de calidad, seguimiento de postventa y servicio de calidad a precios adecuados, para la satisfacer las necesidades del cliente con un respaldo tecnológico y de calidad, logrando al mismo tiempo una rentabilidad para nuestros accionistas».

\section{Visión}

En marzo del 2011 se modificó la visión global producto de la articulación entre la clase de compañía que es Toyota y la que debería ser. Así se llegó a la nueva visión global:

«Recompensada con una sonrisa de nuestros clientes y superando sus expectativas es que Toyota mostrará el camino para el futuro de la movilidad, aportando a las comunidades las formas más seguras y responsables de manejo. A través del compromiso con la calidad, la innovación constante y el respeto por el planeta, el objetivo es superar las expectativas de los clientes y ser recompensados con una sonrisa. Cumplir estos difíciles objetivos solo es posible gracias al talento y pasión con que nuestros colaboradores participan».

\section{Principios}

1. Honrar el contenido y el espíritu de las leyes de todas las naciones del mundo y llevar a cabo actividades de política abierta y justa para ser un buen ciudadano corporativo en todo el mundo.

2. Respetar la cultura y tradiciones de todas las naciones y contribuir al desarrollo económico y social a través de las actividades corporativas en las comunidades locales.

3. Dedicarnos a proveer productos limpios y seguros y contribuir a la mejora en la calidad de vida en todo el mundo por medio de nuestras actividades.

4. Crear y desarrollar tecnología de avanzada y suministrar productos y servicios de primer nivel que satisfagan las necesidades de nuestros clientes en todo el mundo.

5. Promover una cultura corporativa que realce la creatividad individual y el valor del trabajo en equipo, honrando, a la vez, la confianza mutua y el respeto entre los directivos y los empleados.

6. Lograr el crecimiento en armonía con la comunidad global a través de una dirección innovadora.

7. Trabajar con los socios del negocio en la investigación y la creación para obtener un crecimiento a largo plazo y beneficiarnos mutuamente, sin perder de vista las posibilidades de establecer nuevos lazos de negocios (Toyota Argentina s.f.).

\section{Valores}

- Honestidad.

- Lealtad.

- Respeto.

- Responsabilidad.

- Confianza.

\section{Ámbito de operación y marcas}

La corporación Toyota es una empresa global porque tiene operaciones de fabricación y comercialización en todos los continentes: Norteamérica, El Caribe, Latinoamérica, Europa, África, Asia y Oceanía.

\section{Marcas}

Yaris, Prius, Corolla, Avanza, Avensis, Auris.

\section{RESULTADOS Y DISCUSIÓN}

Los conceptos de la distribución comercial, por lo tanto, los canales de distribución y los intermediarios, son aplicables al comercio internacional, con las adecuaciones a los entornos del país en los que operará la empresa.

El tema central de la investigación corresponde al análisis de los canales de distribución en las operaciones del marketing internacional. En este contexto se van a discutir los objetivos planteados en el presente estudio:

* Identificar las distintas estrategias disponibles para diseñar canales de distribución comercial internacional.

* Identificar las variables pertinentes que intervienen en el diseño de un canal distribución comercial internacional que generen ventajas diferenciales.

* Identificar la estrategia de canal de distribución comercial de Toyota que le otorga 
una ventaja diferencial en el mercado internacional frente a sus competidores.

Estrategias disponibles para diseñar canales de distribución comercial internacional.

Pelton, Strutton y Lumplkin (1999) afirman que el punto de vista tradicional es que los flujos del canal empiezan con un fabricante-productor y terminan con un usuario-consumidor. Por lo tanto, el análisis del diseño de canales y de los procesos relacionados generalmente se realizara desde el punto de vista del productor. (p.56)

Se han identificado las siguientes estrategias empleadas para el diseño del canal de distribución de la corporación Toyota, cuyos diseños pueden variar a lo largo de tres dimensiones: (i) número de niveles en el canal, (ii) número de intermediarios que opera en cada nivel, y (iii) tipos de intermediarios en cada nivel.

\section{Numero de niveles en el canal.}

Se estableció que en el país de origen y en el extranjero su canal de distribución es de dos niveles, ya que va del distribuidor oficial a los diversos concesionarios, y estos concesionarios ven cómo colocan el producto en el mercado. En el caso de Perú, el distribuidor oficial es Toyota del Perú y este cuenta con concesionarios y estos concesionarios tienen sus propios puntos de ventas por distritos, $\mathrm{y}$ así llegan al consumidor final

\section{Número de intermediarios en cada nivel.}

Hay tres opciones básicas disponibles: (i) distribución intensiva, (ii) distribución exclusiva, y (iii) distribución selectiva. De la investigación realizada, se observa que Toyota aplica una estrategia de cobertura selectiva por las siguientes consideraciones

- Por la existencia de un alto grado de coordinación entre los miembros del canal, sustentado en la declaración de principios. La misma fue adoptada en 1992 y revisada en 1997, y refleja el tipo de compañía que busca ser manteniendo una unidad en cuanto a su filosofía, valores y metodologías.

- Adopta un sistema vertical de distribución en el modo corporativo mediante la operación de subsidiarias y distribuidores de propiedad total en todo el mundo y contractual porque sus miembros del canal (concesionarios) firman un contrato que establece el periodo de relación comercial (mínimo tres años), de exclusividad territorial y la obligación de comercializar solo las marcas de la fábrica.

- Por el tipo de producto que ofrece y por los estándares que tiene para seleccionar a sus concesionarios.

\section{Tipos de intermediarios en cada nivel.}

Como ya se ha informado, la empresa opera en dos niveles: primer nivel son los mayoristas y en el segundo nivel, los minoristas. Los mayoristas reciben la nomenclatura de "distribuidor", tanto en el país de origen como en los países anfitriones. A los minoristas se les llama "concesionarios". Los concesionarios son de dos tipos: $3 \mathrm{~S}$ y $2 \mathrm{~S}$. La categoría $3 \mathrm{~S}$, además de la función de ventas, ofrece los servicios de postventa tales como mantenimiento, reparación, venta de repuestos y respaldo de garantía de fábrica de los vehículos vendidos por el distribuidor o los concesionarios.

Las variables pertinentes que intervienen en el diseño de un canal distribución comercial internacional y que generan ventajas diferenciales

En muchas empresas grandes, la internacionalización se pude producir de forma relativamente continua de modo que la organización da varios pasos de internacionalización con diversos proyectos simultáneos de expansión en el extranjero, en etapas graduales, durante cierto periodo de tiempo (Hollensen y Arteaga 2010, p. 49).

En el caso de estudio la internacionalización de Toyota ha sido gradual. En la literatura especializada existen varias teorías sobre la internacionalización, para el caso de estudio se deduce que la empresa aplicó el modelo de internacionalización de Upsala, que señala que las empresas tienden a intensificar su compromiso con los mercados extranjeros a medida que aumenta su experiencia; un proceso paulatino de aprender haciendo cuyas variables explicativas son el conocimiento/compromiso con el mercado de la empresa. Esto debe hacerse en pequeños pasos graduales como elegir nuevos mercados geográficos a menor distancia psicológica de los existentes y un modo de entrada con pocos riesgos marginales (p. 106).

En su página web se presenta la información respecto de sus 75 años de existencia (Toyota Motor Corporation s.f. b) y los dividen en tres fases: 
* Tomando el negocio automotriz 1930-1960.

* Entrar en el negocio automotriz 1960-1980.

* Salto adelante como una corporación global 1980-2012.

La etapa de 1930 a 1960 se denomina "tomando el negocio automotriz", ya que la familia Toyoda inicialmente participó en la industria textil, y en septiembre de 1933 toman la decisión de ingresar al negocio automotriz. Cuatro años después, en agosto de 1937, se cristaliza el negocio como Toyota Motor Co. Ltd. En este periodo se consolida las funciones de ventas, en abril de 1950 establecen la organización Toyota Motor Sales Co. Ltd., y en los años siguientes inician sus exportaciones y los primeros acuerdos de distribución en el extranjero.

La etapa de 1960 a 1980 denominada "entrar en el negocio automotriz", se consolida su participación directa en muchos países mediante el establecimiento de su organización Toyota Motor Sales Co. Ltd., y el incremento de acuerdos de distribución en diferentes regiones del mundo; en paralelo instala plantas de producción. También desarrollan distintos modelos de automóviles y camiones, basados en las actividades de I+D, con orientación a la calidad, obteniendo los premios Deming y Calidad Japonesa.

En la etapa de 1980 a 2012, llamada "Salto adelante como una corporación global”, el énfasis es en el desarrollo de las nuevas tecnologías aplicadas al uso de combustibles en los automóviles. Así instalan plantas de producción en las principales regiones del mundo, y amplían su red de distribución a nivel mundial, alcanzando la cifra de 173 distribuidores. Para lograr estos objetivos, en julio de 1982 fusionan las organizaciones Toyota Motor Co. y Toyota Motor Sales, imprimiendo un fuerte impulso a su internacionalización de su operación en el mundo.

\section{Modo de entrada al mercado internacional}

Un modo de entrada en un mercado internacional es un acuerdo institucional necesario para llevar los productos, tecnologías y capital humano de una empresa a un país/mercado extranjero. Los modos de entrada se pueden agrupar en tres categorías básicas: (i) modos de exportación, (ii) modos contractuales, y (iii) modos de inversión. Cada una de estas tiene diferentes grados de control, riesgo y flexibilidad (Cateora y Graham 2006, pp. 415-421).
Como vemos en la cronología de la historia de Toyota, la empresa ha dado pasos graduales en el transcurso de su vida institucional. En su primera etapa de 1930 a 1960 su internacionalización se basó en las exportaciones; de 1960 a 1980 aplicó el modo intermedio (contractuales) y modos de inversión, ya que se instaló en los diferentes países de regiones del mundo estableciendo centros de producción, oficinas de ventas y concesionarios.

\section{Estructura del canal}

Considerando los criterios de control, riesgo y flexibilidad al internacionalizarse, la corporación opera con dos niveles de intermediarios: (i) mayorista, denominado "distribuidor" y (ii) detallista o minorista, denominado "concesionario".

Como se aprecia, la longitud del canal en el extranjero es larga por tener dos niveles de intermediarios y, teniendo en consideración el grado de vinculación existente entre los miembros del canal, se aprecia que el canal de distribución de Toyota asume un sistema vertical de distribución ya que en la vinculación de la fábrica con los intermediarios existe una coordinación muy estrecha, (i) con sus distribuidores establece un sistema vertical corporativo, por cuanto la corporación asume la propiedad de ellos, (ii) con los concesionarios que son entidades independientes, existe una sistema vertical contractual ya que la vinculación de la principal con ellos se consolida mediante la firma de un contrato que establece el periodo de relación comercial, las condiciones asumidas, las normas relacionales y las sanciones previstas en caso de incumplimiento de las clausulas por una de las partes

\section{Estrategia de cobertura del mercado}

La estrategia de cobertura de mercado es de carácter selectiva porque sus automóviles los vende a través de unos pocos distribuidores y concesionarios especializados que le asegura un volumen de ventas superior, tan es así que la marca es el mayor vendedor de automóviles en el mundo. Analistas especializados han informado respecto de la industria automotriz como sigue: «El grupo automovilístico japonés Toyota revalidó en el 2013 el puesto de primer fabricante mundial de automóviles con unas ventas mundiales de 9.98 millones de vehículos, lo que representa un crecimiento del $2 \%$ respecto a 2012 , según datos de la empresa. La corporación estadounidense General Motors ocupó la segunda posición en el ranking de fabricantes mundiales con 9.71 millones de 
vehículos comercializados, lo que representa un crecimiento del $4 \%$ en relación con el ejercicio precedente. El consorcio alemán Volkswagen se situó en tercer lugar, con un volumen total de 9.7 millones de vehículos, incluyendo los vehículos industriales de MAN y Scania. Si se excluyen estas marcas, las entregas de la compañía con sede en Wolfsburgo se elevan a 9.5 millones de unidades, un $4.8 \%$ más» (Autor año).

\section{CONCLUSIONES}

Del estudio se arribó a las siguientes conclusiones:

1. Para cualquier organización empresarial, su filosofía empresarial es la base para una correcta la toma de decisiones con vistas al logro de sus objetivos, sustentados en la declaración de su Misión, Visión y Principios. La razón fundamental para el éxito de Toyota en el mercado global yace en su filosofía corporativa que les ha proporcionado la fuerza de su liderazgo en la industria automotriz.

2. En el proceso de internacionalización, la elección del modo de entrada a un nuevo mercado es una cuestión estratégica clave para las empresas que actúan en los mercados de rápida internacionalización.

3. La distribución comercial desempeña el papel estratégico en el marketing, por ser fuente de ventajas competitivas sostenibles, porque contribuye a la diferenciación y posicionamientos de sus productos y marcas en el mercado respecto de sus competidores.

4. El diseño del canal de distribución (local e internacional) depende de adecuadas decisiones estratégicas en cuanto a su modo de entrada al mercado, configuración de la estructura del canal y las estrategias de cobertura. Para la corporación Toyota, la distribución es fuente de ventaja competitiva sostenible que le otorga poder de mercado en la industria automotriz.

\section{LITERATURA CITADA}

Cateora, Philip y Graham, Jhon, -(2006) - Marketing Internacional, McGraw-Hill, México.

Hollensen, Sved y Arteaga, Jesús, (2010) Estrategias de Marketing Internacional, Person Educacion S.A., Madrid.

Miquel, S., Pacora, F., Lhermie, Ch. y Miquel, M.J. (2008). Distribución Comercial. Sexta edición. ESIC Editorial, Madrid.

Pelton, L.; Strutton, D.; y Lumplkin, J. (1999). Canales de marketing y distribución comercial. McGraw-Hill Interamericana SA., Bogotá.

Toyota Argentina. (s.f.). "Reporte de sustentabilidad año 2013. [En línea]. Fecha de consulta: 22/09/2014. Disponible en: <http:www. toyotanet.com.ar>.

Toyota Motor Corporation. (s.f. a) Sitio web global. Fecha de consulta: 20/08)2014. Disponible en: <http://www.toyota-global.com/>.

Toyota Motor Corporation. (s.f. b). "75 años de Toyota". Fecha de consulta: 27/08/2014. Disponible en: <http://www.toyota-global. com/company/history_of_toyota/75years/ index.html>.

Vásquez, R., y Trespalacios, J. (2006). Estrategias de Distribución Comercial. International Thomson Editores Spain Paraninfo S.A., Madrid. 